\title{
Floraciones algales nocivas durante el periodo 2008-2010 en el Golfo de Nicoya, Costa Rica
}

\section{Harmful algal blooms during 2008-2010 in the Gulf of Nicoya, Costa Rica}

\author{
Emilia Calvo Vargas ${ }^{1 *}$, Karen Berrocal Artavia ${ }^{1}$ y Jorge Boza Abarca ${ }^{1}$
}

\begin{abstract}
RESUMEN
En este estudio se analizaron los eventos de floraciones algales nocivas (FAN) en cuatro estaciones de muestreo fijas en el Golfo de Nicoya durante el periodo de enero de 2008 a diciembre de 2010. Se recolectaron muestras de agua a 1 y $5 \mathrm{~m}$ de profundidad durante cada evento de FAN. Además, se midieron in situ los parámetros fisicoquímicos: profundidad del disco de Secchi, temperatura, salinidad, oxígeno disuelto y el porcentaje de saturación de oxígeno del agua. Los datos de radiación solar y precipitación fueron suministrados por el Instituto Meteorológico Nacional de Costa Rica. Se determinaron las concentraciones de las microalgas responsables y las especies asociadas. Se registraron 11 eventos, de los cuales el $72.7 \%$ se presentaron en la época lluviosa y un $27.3 \%$ en la época seca, la mayoría de éstos, durante el fenómeno atmosférico de La Niña. Las especies responsables fueron los dinoflagelados Alexandrium monilatum, Levanderina fissa, Cochlodinium polykrikoides y Prorocentrum minimum, la diatomea Skeletonema costatum y la cianobacteria Trichodesmium erythraeum. Las especies asociadas que se registraron con mayor frecuencia fueron los dinoflagelados Dinophysis caudata, Prorocentrum micans, Tripos fusus, Tripos furca y Protoperidinium conicum. Las FAN en el Golfo de Nicoya fueron más frecuentes durante la época lluviosa, probablemente debido a los aportes fluviales al sistema estuarino de los ríos Tempisque, Barranca y Grande de Tárcoles. Esa estacionalidad, en conjunto con las alteraciones provocadas por eventos atmosféricos globales como La Niña y El Niño, determinó la formación de FAN en el golfo durante el periodo de estudio.
\end{abstract}

Palabras claves: Floraciones algales nocivas, microalgas responsables, condiciones ambientales, El Niño y La Niña, Golfo de Nicoya.

\begin{abstract}
Harmful Algal Blooms (HABs) events were analyzed in this study at four monitoring stations in the Gulf of Nicoya between January 2008 and December 2010. Water samples were collected at 1 and $5 \mathrm{~m}$ deep during each HAB event. Furthermore, the following physicochemical parameters were measured in situ: Secchi disk depth, temperature, salinity, dissolved oxygen, and saturation percentage of oxygen in water. Radiation and precipitation data was provided by the National Meteorological Institute of Costa Rica. The concentration of dominant microalgae and associated species was determined. A total of 11 events were recorded, $72.7 \%$ during rainy season and $27.3 \%$ during dry season, most of them during the La Niña atmospheric phenomenon. Dominant species included the following dinoflagellates; Alexandrium monilatum, Levanderina
\end{abstract}

Estación de Biología Marina Juan Bertoglia Richards, Escuela de Ciencias Biológicas, Universidad Nacional, Puntarenas, Costa Rica.emilia.calvo.vargas@una.cr*y ecalvo09@gmail.com

Recibido: 29 de setiembre de 2015

Corregido: 18 de enero de 2016

Aceptado: 21 de enero de 2016

DOI: http://dx.doi.org/10.15359/revmar.8-1.9

Rev. Mar. Cost. ISSN 1659-455X. Vol. 8 (1): 129-149, Enero-Junio 2016. 
fissa, Cochlodinium polykrikoides and Prorocentrum minimum, as well as diatom Skeletonema costatum and cyanobacterium Trichodesmium erythraeum. The most frequent associated species were dinoflagellates Dinophysis caudata, Prorocentrum micans, Tripos fusus, Tripos furca and Protoperidinium conicum. HABs in the Gulf of Nicoya were more frequent during the rainy season, probably due to the contributions of the Tempisque, Barranca and Grande de Tárcoles rivers to the estuarine system. This seasonality, together with the alterations caused by El Niño and La Niña atmospheric phenomena, determined the development of HABs in the Gulf of Nicoya during the study period.

Keywords: Harmful algal blooms, dominant microalgae, environmental conditions, E1 Niño, La Niña, Gulf of Nicoya.

\section{INTRODUCCIÓN}

El fitoplancton es un constituyente esencial de la cadena alimentaria marina y representa el $40 \%$ de la productividad primaria total mundial (Narayana et al. 2014), en las regiones costeras vive en un ambiente dinámico, con frecuentes y repentinos cambios en las condiciones ambientales. Los parámetros abióticos (turbidez, intensidad de luz, salinidad y disponibilidad de los nutrientes) pueden variar en niveles espaciales muy locales en la columna de agua. Las interacciones bióticas entre las microalgas y los fitófagos potenciales o patógenos influyen en el crecimiento de la población de determinadas especies de microalgas (Alpermann et al. 2010).

Las floraciones algales nocivas (FAN) son conocidas como mareas rojas, debido a la coloración rojiza del agua, se desarrollan en áreas costeras eutrofizadas, causando un gran daño a los organismos marinos, incluyendo mortalidad de peces y organismos bentónicos, e intoxicaciones por moluscos (Choi et al. 2014). En las últimas décadas, el impacto de las FAN sobre los ecosistemas, las pesquerías, el turismo y la salud pública se ha intensificado, permitiendo una mayor atención científica y regulatoria al desarrollo de nuevas tecnologías para la investigación y el manejo de éstas (Anderson et al. 2012).

La tendencia del incremento de las FAN es atribuida a la eutrofización costera y al cambio climático, mientras que el crecimiento repentino y la formación de las FAN, en general, son el resultado de la capacidad que tienen las especies responsables para utilizar eficientemente los nutrientes del ambiente -en exceso o limitados- para absorber la energía lumínica y disuadir el pastoreo, cada floración se puede desencadenar por un conjunto de factores ambientales (Anderson et al. 2012; Zhuang et al. 2015).

Existe un número amplio de especies de microalgas responsables de los eventos de FAN (Taylor et al. 2014), entre ellas, varias especies de diatomeas, dinoflagelados, rafidófitas, primnesiófitas, cianobacterias, pelagófitas y silicoflagelados (Basti et al. 2015). En general, en la Bahía de La Paz, Golfo de California, se han reportado FAN de dinoflagelados como Cochlodinium polykrikoides en el 2000 y el 2001 causando mortalidades de peces (Gárate-Lizárraga et al. 2004), y de Gonyaulax polygramma en el 2004, que produjo una floración frente 
a la Isla Espíritu Santo en la misma área, causando mortalidad de peces por falta de oxígeno, ya que no es tóxica (GárateLizárraga et al. 2006). Entre el 2005 y el 2011 ocurrieron siete floraciones de Trichodesmium erythraeum y T. thiebautii, sin causar ningún efecto sobre la biota marina de la zona (Gárate-Lizárraga \& Muciño-Márquez, 2012); en el 2012, se repite una proliferación de $C$. polykrikoides, esta vez sin causar mortalidad de peces (Gárate-Lizárraga, 2013). En el 2013, se registró en la Bahía de Acapulco la primera FAN de Gyrodinium instriatum, sin causar mortalidades en otros organismos (Gárate-Lizárraga et al. 2013), y en el 2014 en La Paz, Golfo de California, se observaron las proliferaciones de L. fissa y Polykrikos hartmannii (Gárate-Lizárraga, 2014).

En la costa pacífica de Costa Rica, desde el 2002 varias FAN de C. polykrikoides han sido reportadas produciendo altas mortalidades de peces y efectos nocivos en la población costera, y se han relacionado con el aumento en los vientos (Vargas-Montero et al. 2006). Vargas-Montero et al. (2008) reportaron al menos 13 taxones de dinoflagelados productores de FAN para el periodo 1979-2005, destacándose las FAN de Pyrodinium bahamense, Alexandrium monilatum y C. polykrikoides.

En el Golfo de Nicoya, el grupo principal que ha formado FAN históricamente son los dinoflagelados, en algunos casos las diatomeas y en algunas ocasiones las cianobacterias (Calvo et al. 2005; Vargas-Montero et al. 2008).

El Golfo de Nicoya, situado en el litoral Pacífico de Costa Rica $\left(9^{\circ}\right.$ $48^{\prime} 0^{\prime \prime} \mathrm{N}, 84^{\circ} 48^{\prime} 0^{\prime \prime} \mathrm{W}$ ), es un estuario altamente productivo, donde el fitoplancton juega un papel relevante al soportar grandes comunidades de organismos marinos de importancia comercial. La extracción de productos de la pesca y la acuicultura representan el medio de subsistencia de muchas comunidades del golfo, en particular aquellas de escasos recursos económicos. Es por ello, que el estudio del fitoplancton es vital al influir éste de forma directa en el estado de las pesquerías del golfo. Con base en lo anterior, el objetivo de este estudio fue registrar y analizar las FAN durante el periodo de enero de 2008 a diciembre de 2010 para determinar las condiciones ambientales que favorecen estos eventos y comprender mejor sobre su presencia en el golfo y, de esta manera, prevenir y/o mitigar sus impactos.

\section{MATERIALES Y MÉTODOS}

\section{Descripción del área de estudio}

Este estudio fue realizado en el Golfo de Nicoya (Fig. 1), el cual es considerado un estuario con ecosistemas litorales y ríos con una riqueza natural y económica de gran importancia para la costa pacífica de Costa Rica (Castro \& León, 1998). Sus dimensiones son de aproximadamente $80 \mathrm{~km}$ de largo, $50 \mathrm{~km}$ de ancho y con un área de $1550 \mathrm{~km}^{2}$ (Kress et al. 2001). Su parte interna abarca desde la línea imaginaria entre Puntarenas y la Isla San Lucas, hasta la desembocadura del río Tempisque, zona costera caracterizada por bosques 
de manglar y profundidades bajas (20 $\mathrm{m})$. La parte externa incluye el área hasta la desembocadura de los ríos Grande de Tárcoles y Barranca, cuyo litoral está formado por playas rocosas y arenosas, y profundidades de hasta 100 m (Calvo, 2002; 2012; Calvo Vargas et al. 2014).

Durante el periodo de enero de 2008 a diciembre de 2010, cuatro estaciones fueron monitoreadas cada dos semanas regularmente, y cuando se presentaron los eventos de FAN se aumentó la frecuencia del muestreo. La ubicación geográfica de las estaciones de muestreo fue la siguiente: Isla Pajarita $\left(10^{\circ} 5^{\prime} 30^{\prime \prime} \mathrm{N}\right.$ y $\left.84^{\circ} 59^{\prime} 80^{\prime \prime} \mathrm{O}\right)$ en la parte interna del golfo, Muelle de Puntarenas $\left(9^{\circ} 58^{\prime} 25^{\prime \prime} \mathrm{N}\right.$ y $84^{\circ} 49^{\prime} 76^{\prime}$ " O), Bahía Caldera ( $9^{\circ} 54^{\prime} 90^{\prime \prime} \mathrm{N}$ y $84^{\circ}$ 43' 40" O) y Tárcoles ( $9^{\circ} 44^{\prime} 60^{\prime \prime} \mathrm{N} \mathrm{y}$ 84 38' 70" O) (Fig. 1).

Para el análisis de las FAN, en las cuatro estaciones de muestreo, se recolectaron muestras de agua a 1 y $5 \mathrm{~m}$ de profundidad utilizando una botella oceanográfica Niskin de $5 \mathrm{~L}$ de capacidad. Para el análisis cualitativo, se realizó un arrastre vertical con una red de $20 \mu \mathrm{m}$ de abertura de malla. Además, se midieron in situ a 1 y 5 $\mathrm{m}$ de profundidad, la profundidad del disco de Secchi (m), la temperatura del agua $\left({ }^{\circ} \mathrm{C}\right)$, la salinidad (UPS), el oxígeno disuelto $\left(\mathrm{mg} \mathrm{L}^{-1}\right)$ y el porcentaje de saturación con una sonda multiparamétrica (modelo YSI Incorporated, YSI 556 MPS). La concentración celular en las muestras de agua se determinó por duplicado $(\mathrm{n}=2)$, utilizando cámaras de conteo
Sedgewick-Rafter. En el laboratorio y empleando un microscopio invertido (Nikon Eclipse TE 2000-S), se identificaron las especies de microalgas y la concentración celular por especie y muestra $\left(\right.$ Cél L $\left.{ }^{-1}\right)$. Los resultados se complementaron con datos de precipitación $(\mathrm{mm})$ y radiación solar $\left(\mathrm{MJ}\left(\mathrm{m}^{2}\right)^{-1}\right)$ (Calvo Vargas et al. 2014), que fueron suministrados por el Instituto Meteorológico Nacional de Costa Rica. Los eventos fueron monitoreados desde su inicio hasta que las concentraciones algales volvieran a la normalidad en el Golfo de Nicoya.

Los datos obtenidos fueron agrupados en cuadros caracterizando los eventos en cuanto a parámetros fisicoquímicos y meteorológicos. Para determinar las microalgas acompañantes que fueron más frecuentes y relacionadas con las diferentes microalgas que provocaron las FAN, se realizó un análisis de conglomerados jerárquicos con el programa estadístico SPSS versión 17.0 (SPSS, 2006).

\section{RESULTADOS}

Se observaron 11 eventos de FAN en diferentes estaciones de muestreo: ocho en la época lluviosa (mayo-noviembre) y tres en la época seca (diciembre-abril), representando el $72.7 \%$ y $27.3 \%$, respectivamente. Ocho de los eventos de FAN ocurrieron durante las condiciones de La Niña y tres condiciones de El Niño (Cuadro 1).

La concentración mínima registrada en una FAN de Alexandrium monilatum fue de 33450 Cél L $^{-1}$, en noviembre de 2009, en laépocalluviosa 


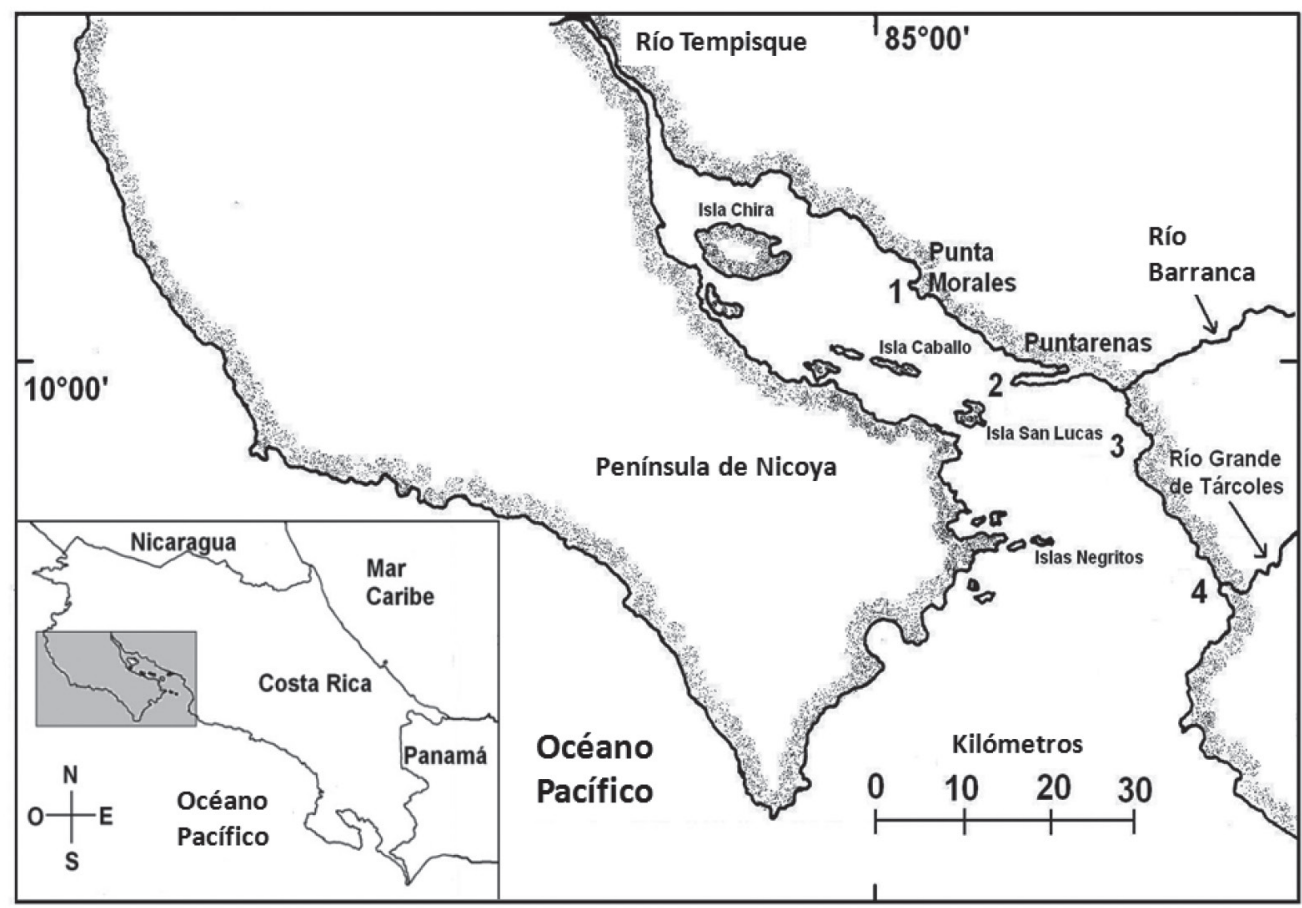

Fig. 1. Área de estudio. Los números indican la ubicación de las estaciones de muestreo en el Golfo de Nicoya: (1) Isla Pajarita, (2) Muelle de Puntarenas, (3) Bahía Caldera, (4) Tárcoles. Fuente: Calvo Vargas et al. (2014)

Fig. 1. Study area. Numbers indicate the sampling stations in the Gulf of Nicoya: (1) Isla Pajarita, (2) Muelle the Puntarenas, (3) Bahía Caldera, (4) Tárcoles. Source: Calvo Vargas et al. (2014)

caracterizada por ser El Niño. Mientras que en octubre de 2010, en la estación Muelle de Puntarenas, se determinó la concentración máxima de 3371 150 Cél L $^{-1}$, siendo la época lluviosa, pero en condiciones de La Niña. Esta FAN también fue contabilizada en la estación Bahía Caldera (1 544400 Cél $\mathrm{L}^{-1}$ ), desplazándose principalmente en la parte externa del golfo. La profundidad del disco de Secchi varió de 1.50 a $2.75 \mathrm{~m}$, la temperatura del agua de 27.40 a $28.36^{\circ} \mathrm{C}$, la salinidad de 25.46 a 31.46 UPS, el oxígeno disuelto de 6.26 a $9.40 \mathrm{mg} \mathrm{L}^{-1}$, la saturación de oxígeno de 91.80 a $140.45 \%$, la precipitación de 51.70 a $570.60 \mathrm{~mm}$ y la radiación de 1.63 a $1.84 \mathrm{MJ}\left(\mathrm{m}^{2}\right)^{-1}$ (Cuadro 1).

Skeletonema costatum también fue registrada en la parte externa del golfo en tres de las estaciones muestreadas (Tárcoles, Bahía Caldera y Muelle de Puntarenas), con concentraciones mínimas de 1111950 Cél L $\mathrm{L}^{-1}$ (noviembre de 2010, Muelle de Puntarenas), durante la época lluviosa y el fenómeno de La Niña, mientras que la máxima concentración de 6545000 Cél L $\mathrm{L}^{-1}$ ocurrió en la estación Tárcoles 


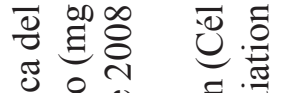
苍造造

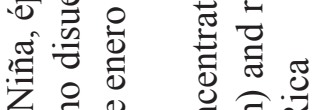

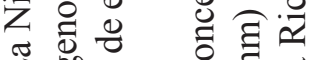

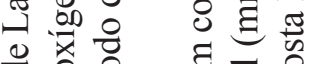
ㅇํㅇ तेक्षे

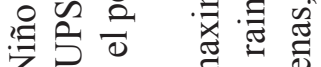

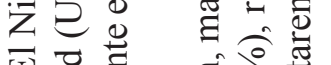

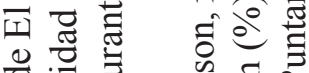

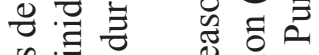

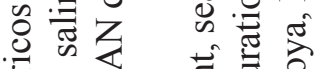

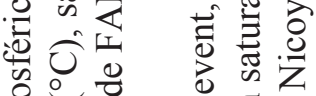

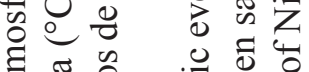

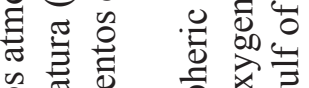

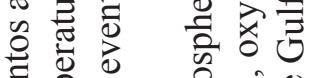

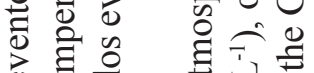
5. 흐응

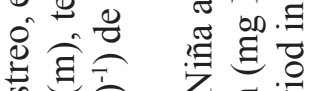
해을

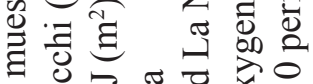

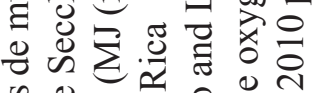

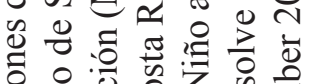

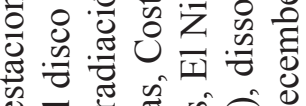

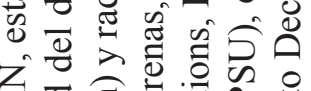

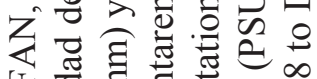

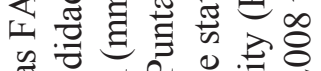

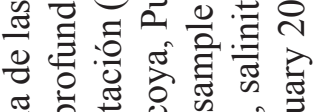

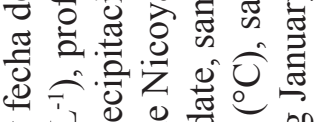

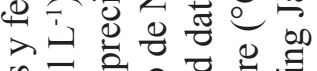
象 可

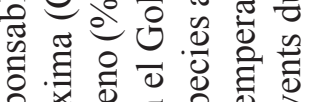
然.

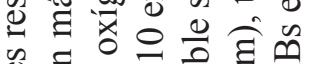

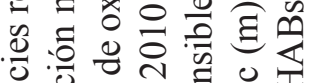

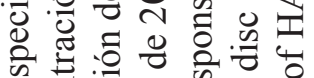

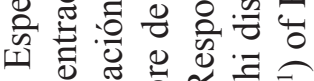
-

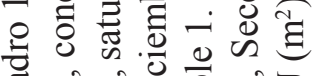

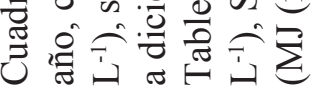

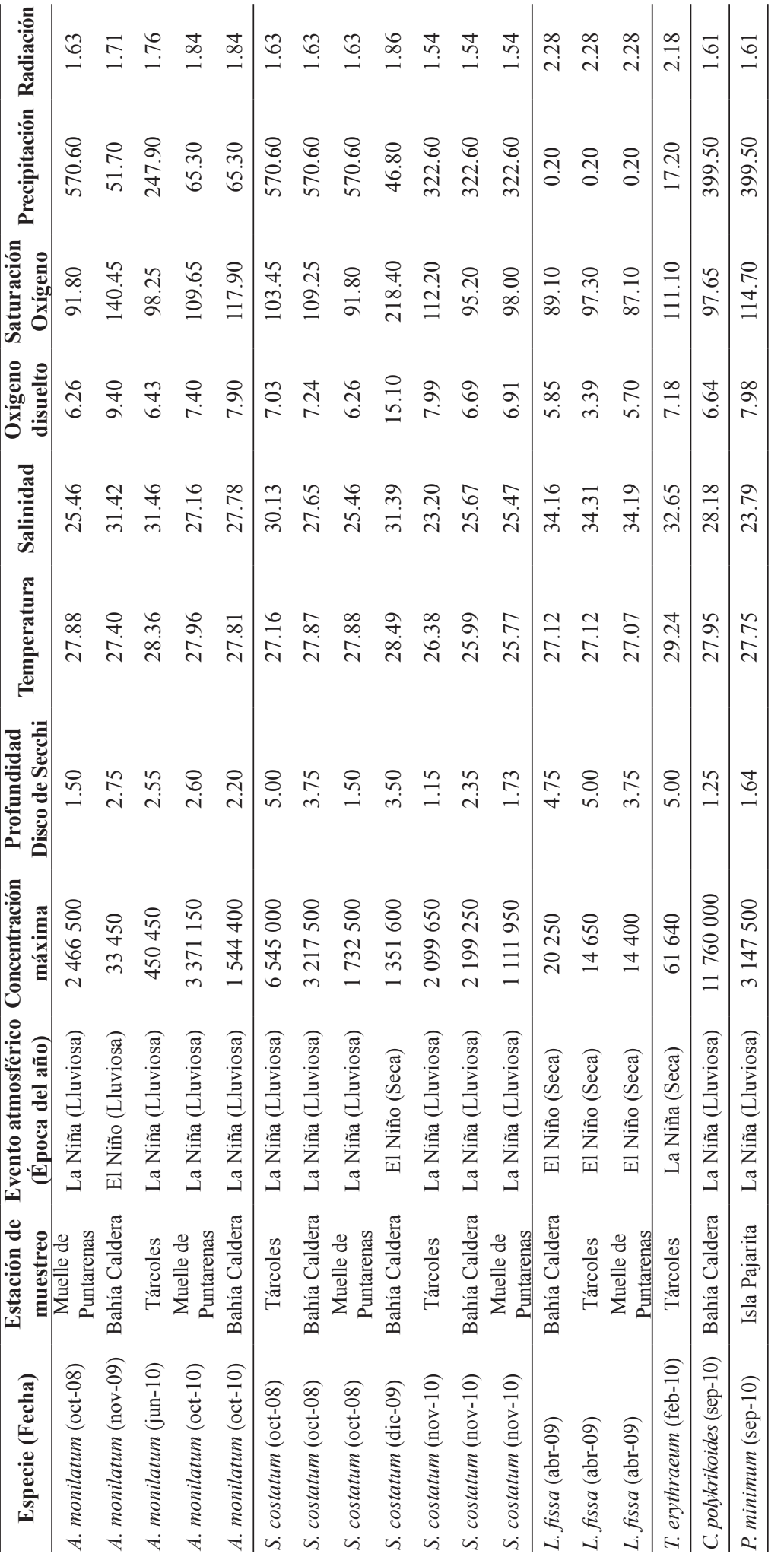


en octubre de 2008, durante la época lluviosa y en condiciones de La Niña. Solo se dio un evento de FAN durante la época seca (El Niño) en diciembre de 2009 en la estación Bahía Caldera. La profundidad del disco de Secchi varió de 1.15 a $5.00 \mathrm{~m}$, la temperatura del agua estuvo entre 25.77 y $28.49^{\circ} \mathrm{C}$, mientras que la salinidad varió de 23.20 a 31.39 UPS, el oxígeno disuelto osciló entre 6.26 y $15.10 \mathrm{mg} \mathrm{L}^{-1}$, la saturación de oxígeno entre $91.80 \mathrm{y}$ $218.40 \%$, la precipitación entre 46.80 y $570.60 \mathrm{~mm}$ y la radiación entre 1.54 y $1.86 \mathrm{MJ}\left(\mathrm{m}^{2}\right)^{-1}$ (Cuadro 1 ).

La especie Levanderina fissa registró en abril de 2009 un solo evento que cubrió las estaciones en la parte externa del golfo, con una concentración mínima de 14400 Cél $\mathrm{L}^{-1}$ en el Muelle de Puntarenas, y una máxima de 20250 Cél L $^{-1}$ en Bahía Caldera, durante la época seca y el fenómeno de El Niño. Los intervalos de las variables fisicoquímicas tuvieron los siguientes valores: la profundidad del disco de Secchi varió de 3.75 a $5.00 \mathrm{~m}$, la temperatura del agua estuvo entre 27.07 y $27.12^{\circ} \mathrm{C}$, la salinidad entre 34.16 y 34.31 UPS, el oxígeno disuelto en un intervalo de 3.39 a 5.85 mg L L ${ }^{-1}$ la saturación de oxígeno de 87.10 a $97.30 \%$, la precipitación en $0.20 \mathrm{~mm}$ y, por último, la radiación en $2.28 \mathrm{MJ}\left(\mathrm{m}^{2}\right)^{-1}$ (Cuadro 1).

Trichodesmium erythraeum se observó formando FAN una sola vez en febrero de 2010 en Tárcoles, con una concentración de $61640 \mathrm{Cél} \mathrm{L}^{-1} \mathrm{y}$ durante La Niña en la época seca. La profundidad del disco de Secchi fue de $5.00 \mathrm{~m}$, la temperatura del agua de $29.24^{\circ} \mathrm{C}$, la salinidad de 32.65 UPS, el oxígeno disuelto de $7.18 \mathrm{mg} \mathrm{L}^{-1}$, la saturación de oxígeno de $111.10 \%$, con baja precipitación $(17.20 \mathrm{~mm})$, y la radiación alta de $2.18 \mathrm{MJ}\left(\mathrm{m}^{2}\right)^{-1}$ (Cuadro 1).

Cochlodinium polykrikoides formó solamente un evento de FAN en condiciones de La Niña y en la época lluviosa, alcanzando una concentración pico de 11760000 Cél L $^{-1}$ en setiembre de 2010 en la estación Bahía Caldera (Cuadro 1). Los intervalos de los parámetros fisicoquímicos fueron los siguientes: la profundidad del disco de Secchi varió de 0.5 a $2.0 \mathrm{~m}$, la temperatura del agua de 26.68 a $28.70^{\circ} \mathrm{C}$, la salinidad de 27.99 a 31.48 UPS, el oxígeno disuelto de 6.40 a $7.80 \mathrm{mg} \mathrm{L}^{-1}$, la saturación de oxígeno de 96.70 a $116.40 \%$, la precipitación de 261.00 a $399.70 \mathrm{~mm}$ y la radiación de 1.61-1.79 MJ $\left(\mathrm{m}^{2}\right)^{-1}$.

Por último, Prorocentrum minimum fue la única especie que formó FAN en la parte interna del golfo, durante el fenómeno de La Niña y en la época lluviosa. Se registró una concentración de 3147500 Cél L $^{-1}$ en setiembre de 2010, en la estación Isla Pajarita. Las variables fisicoquímicas mostraron los siguientes valores: la profundidad del disco de Secchi fue de $1.64 \mathrm{~m}$, la temperatura del agua de $27.75^{\circ} \mathrm{C}$, la salinidad de 23.79 UPS, el oxígeno disuelto de $7.98 \mathrm{mg} \mathrm{L}^{-1}$, la saturación de oxígeno de $114.70 \%$, la precipitación de $399.50 \mathrm{~mm}$ y la radiación de 1.61 $\mathrm{MJ}\left(\mathrm{m}^{2}\right)^{-1}$ (Cuadro 1).

Las especies acompañantes observadas con mayor frecuencia (Fig. 
2) durante los eventos de FAN fueron 39 , pertenecientes a diferentes grupos de microalgas y géneros. Con base en esta información, se realizó un análisis de conglomerados jerárquicos, utilizando la presencia o ausencia de estas microalgas en las FAN (Fig. 3), lo que permitió determinar las especies acompañantes que estuvieron presentes en todos o la mayoría de los eventos de FAN. Tripos furca se encontró presente en todos los eventos de FAN independientemente de la especie responsable de éstos. Prorocentrum micans, Tripos fusus, Dinophysis caudata, Protoperidinium conicum y Dictyocha fibula fueron las especies que más acompañaron a las especies formadoras de FAN, mientras que Lingulodinium polyedrum, Protoperidinium pellucidum, Protoperidinium divergens, Alexandrium sp. y Tripos pentagonum, solo estuvieron presentes en uno de los eventos de FAN registrados en los tres años (Figs. 2 y 3 ).

\section{DISCUSIÓN}

El género Alexandrium incluye algunos de los dinoflagelados más tóxicos (Hansen \& Moestrup, 1998), producen neurotoxinas del grupo de las saxitoxinas (Lim \& Ogata, 2005), cuya toxicidad puede variar entre las especies, debido a su composición y contenido (Anderson et al. 1990). Las FAN del dinoflagelado $A$. monilatum han sido asociadas a la mortalidad de peces e invertebrados y se ha demostrado su efecto nocivo en homeotermos (May et al. 2010).

En este estudio, las FAN de $A$. monilatum ocurrieron una vez cada año, pero en el 2010 estuvieron presentes en tres de las cuatro estaciones muestreadas, todas ubicadas en la parte externa del golfo. La expansión y el incremento en sus concentraciones celulares son indicativos de que las condiciones en el golfo favorecen su proliferación. Según Da-Zhi et al. (2006), la incidencia de las floraciones de Alexandrium han aumentado considerablemente en las últimas décadas en el ámbito mundial.

Las FAN de A. monilatum en elgolfo se vieron favorecidas por temperaturas de 27.4 a $28.36^{\circ} \mathrm{C}$ y salinidades entre 25.46 y 31.46 UPS. En el evento del 2005 , la temperatura fue de 30 a $31.5^{\circ} \mathrm{C}$ y la salinidad de 29 a 30 UPS (Calvo et al. 2005), condiciones diferentes a las encontradas en este estudio con respecto a la temperatura, no así en relación con la salinidad, mientras que todos los eventos sucedieron en la época lluviosa. En experimentos de laboratorio se determinó la tasa máxima de crecimiento a una temperatura de $31^{\circ} \mathrm{C}$ y a salinidades superiores de 15 UPS, lo cual indica que las proliferaciones parecen estar restringidas a aguas costeras y es improbable que se presenten en aguas con temperatura menor a $25^{\circ} \mathrm{C}$ y salinidades por debajo de 15 UPS (Juhl, 2005), condiciones que no caracterizan la parte interna del golfo, en la cual no se presentó este dinoflagelado.

En el Golfo de Nicoya, este dinoflagelado fue un componente frecuente de lasfloraciones algales quese formaron (Víquez \& Hargraves, 1995), sin embargo, en el 2005 se registra por primera vez como la especie responsable 


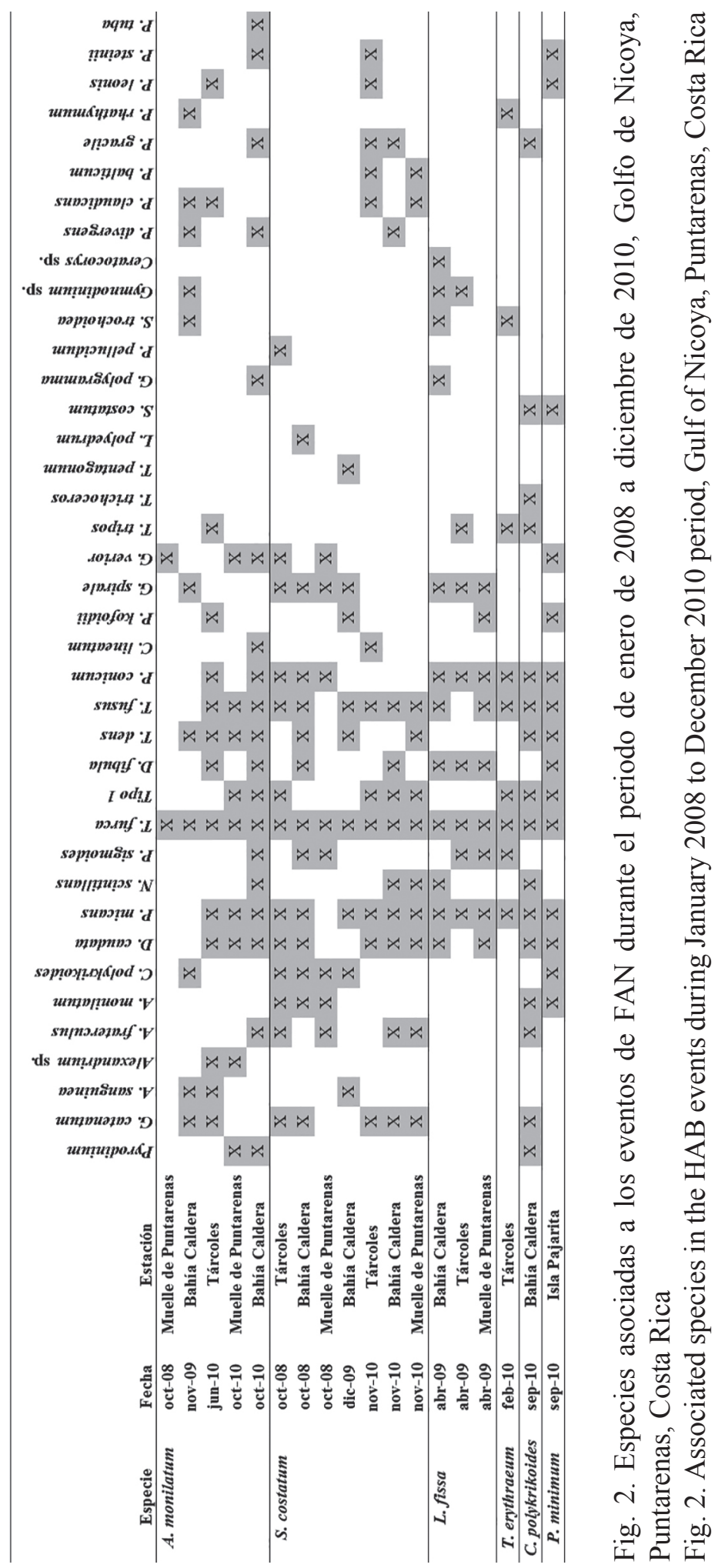


Escala distancia conglomerado

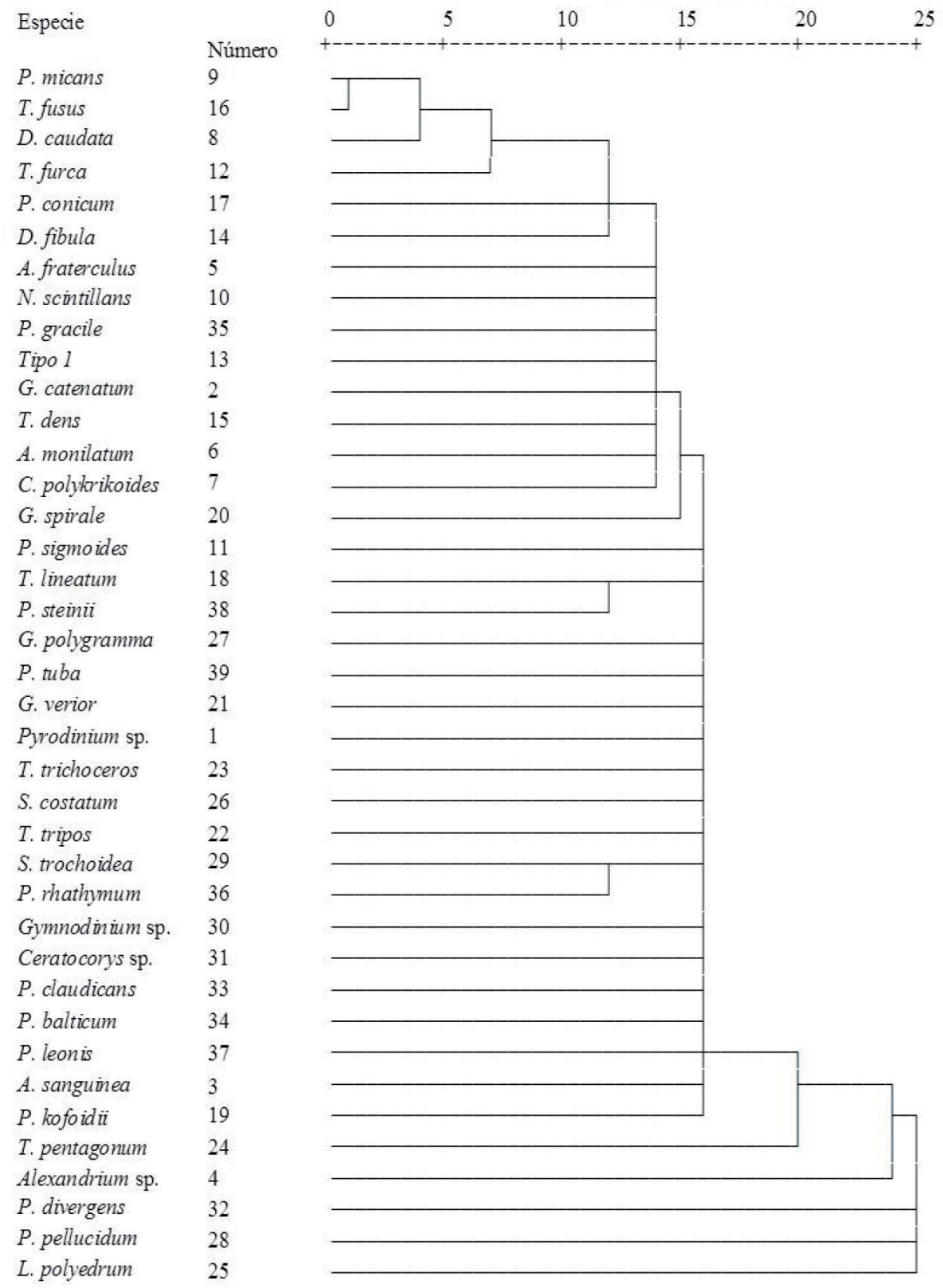

Fig. 3. Análisis de conglomerados jerárquicos para las especies asociadas a los eventos de FAN durante el periodo de enero de 2008 a diciembre de 2010, Golfo de Nicoya, Puntarenas, Costa Rica

Fig. 3. Hierarchical cluster analysis to associated species in the HAB events during January 2008 to December 2010 period, Gulf of Nicoya, Puntarenas, Costa Rica 
de una floración, acompañada por Pyrodinium bahamense var. compressum, Gymnodinium catenatum, Gymnodinium splendens (Akashiwo sanguinea) y Cochlodinium catenatum (C. polykrikoides), además de algunas especies de Ceratium y Prorocentrum (Calvo et al. 2005), mientras que en este estudio se lograron observar 31 especies acompañantes (Fig. 2). Desde el 2005 hasta la fecha $A$. monilatum ha formado cada año floraciones en el golfo, con la excepción del 2011.

La diatomea céntrica $S$. costatum ha sido considerada una de las más ampliamente distribuidas del fitoplancton marino-costero (Sarno et al. 2007) y estuarino (Huo \& Shu, 2005); se considera que es euritérmica y eurihalina, encontrándose sobre todo en aguas ricas en nutrientes. Estudios realizados en la plataforma continental de Long Island (EE. UU.) determinaron una división celular alta en dependencia de la temperatura y la iluminación (Castellví, 1963).

En este estudio, las floraciones de $S$. costatum se observaron en intervalos de temperaturas de 25.77 a $28.49^{\circ} \mathrm{C}$ y salinidades de 23.20 a 31.39 UPS durante los meses de alta precipitación y al final de la época lluviosa. En 2009 se registraron en diciembre, cuando la precipitación había disminuido considerablemente (Calvo, 2012; Calvo Vargas et al. 2014). En la Bahía Jiaozhou de China, la ocurrencia de $S$. costatum estuvo asociada a lluvias fuertes y aguas cálidas con salinidades bajas y eutrofización. La temperatura fue de 25.6 a $28.0^{\circ} \mathrm{C}$ y la salinidad de
30.31 a 30.78 UPS, con alta saturación de oxígeno (Huo \& Shu, 2005). En Utría (Pacífico colombiano), $S$. costatum prolifera en salinidades bajas y aportes de agua continental. Una situación similar con abundancias altas se registró en los manglares del Golfo de Bengala, la India (Peña \& Pinilla, 2002).

Las FAN de $S$. costatum tienen porcentajes muy altos de dominancia específica (93.38-99.75\%) como producto de condiciones ambientales aptas para su desarrollo y probablemente por alelopatía. Yamasaki et al. $(2009 ; 2010)$ realizaron estudios con $S$. costatum en cultivo e in situ, y concluyeron que la diatomea produjo sustancias aleloquímicas con una fuerte actividad lítica en dependencia de la especie objetivo y la concentración celular. Yamaguchi et al. (2011) reafirman que la alelopatía y el contacto celular podrían estar involucrados para que ciertas especies lleguen a ser dominantes en una comunidad. Anteriormente, en Costa Rica esta especie no había sido reportada como formadora de FAN (Vargas-Montero et al. 2008), sin embargo, en el periodo de este estudio apareció durante los tres años acompañada de 28 especies (Fig. 2).

En este estudio se observó un evento de L. fissa en abril de 2009 en tres estaciones ubicadas en la parte externa del golfo, en la época seca y durante un evento de El Niño. $L$. fissa es un dinoflagelado desnudo, fotosintético (Uchida et al. 1997), con una distribución mundial, y no se ha 
asociado a la producción de toxinas, pero sí a situaciones de anoxia en el fondo. La distribución espacial de sus floraciones puede ser muy amplia: en Ecuador abarcó $50 \mathrm{~km}$ a lo largo de la costa norte del golfo de Guayaquil y en China cubrió $150-200 \mathrm{~km}^{2}$ en el estuario Zhujiang y $500 \mathrm{~km}^{2}$ en la Bahía Chi. Esos eventos causaron daños muy serios a los productos pesqueros (Nagasoe et al. 2010). Nagasoe et al. (2006) determinaron que el dinoflagelado puede crecer en temperaturas entre 15 y $30^{\circ} \mathrm{C}$ y salinidades entre 5 y 40 UPS. La tasa óptima de crecimiento la obtuvieron a $20-30^{\circ} \mathrm{C}$ y $10-35$ de salinidad. Las mediciones in situ de la temperatura y la salinidad del agua durante dos floraciones muy intensas en el 2002 y el 2003 en el puerto pesquero de Hakozaki, Japón, también ocurrieron entre esos intervalos. En Costa Rica, L. fissa solo había sido reportado en el 2004 con una sola especie acompañante (A. monilatum) (VargasMontero et al. 2008), mientras que en este estudio la especie estuvo acompañada por 15 especies.

Mundialmente, las cianobacterias causan problemas debido a la eutrofización de las aguas superficiales, en particular por el uso de fertilizantes y abonos en la agricultura, y la descarga de aguas residuales. También se ha visto que el calentamiento del clima influye en su proliferación (Ibelings et al. 2014).

Las cianobacterias del género Trichodesmium suelen formar floraciones en aguas tropicales y subtropicales tranquilas (Negri et al.
2004; Siqueira et al. 2006; Carvalho et al. 2008; Kranz et al. 2010; GárateLizárraga \& Muciño-Márquez, 2012), en estuarios y aguas costeras bajo eutrofización antropogénica. El decaimiento de las floraciones puede provocar condiciones de anoxia y mortalidad de organismos, como fueron los casos de las ostras en la India (Thillairajasekar et al. 2009), y de peces y camarones en Tailandia y Australia (Negri et al. 2004). La especie $T$. erythraeum es una de las más comunes en formar floraciones, ampliamente estudiada por su papel en los ciclos biogeoquímicos y su importancia ecológica (Narayana et al. 2014).

Durante este estudio, se observó un evento de T. erythraeum (febrero de 2010) con temperatura y salinidad altas $\left(29.24^{\circ} \mathrm{C}\right.$ y 32.65 UPS), valores que coinciden con las observaciones de Carvalho et al. (2008), quienes registraron temperaturas entre 27 y $29^{\circ} \mathrm{C}$ y salinidades de 33.1 a 35.2 UPS, en la costa sureste brasileña, y Siqueira et al. (2006) en Paraná, Brasil, donde la cianobacteria sobrevivió en condiciones ambientales relativamente inhóspitas gracias a su capacidad de fijar nitrógeno y realizar la fotosíntesis a altas intensidades de luz. En algunas áreas floreció cerca de la boca de un río del sistema estuarino de Paranaguá, lo que demuestra su preferencia por aguas ricas en nutrientes (Siqueira et al. 2006), situación que coincide con el registro en este estudio en la estación de muestreo cercana a la desembocadura del río Grande de Tárcoles, el cual aporta al golfo nutrientes provenientes del Valle Central. León et al. (1998-1999) señalan que 
durante la época seca en la parte externa del golfo entran vientos muy fuertes provenientes del Caribe (conocidos como "nortes"), provocando que la reserva de nutrientes, que se encuentran en las capas profundas, alcancen las aguas superficiales iluminadas y aumenten la producción de nuevo material vegetal.

La FAN de T. erythraeum es similar a la descrita por Satpathy et al. (2007) en las aguas costeras al este de la India (Kalpakkam), la cual tuvo una duración de un día, alterando las propiedades fisicoquímicas y la estructura de la comunidad fitoplanctónica. Los rangos fueron de 28.1 a $30.3^{\circ} \mathrm{C}$ y de 31.58 a 33.54 UPS y la concentración del oxígeno disuelto fue de 5.7 a $7.1 \mathrm{mg} \mathrm{L}^{-1}$.

En este estudio, la FAN de $T$. erythraeum se presentó en la época seca (febrero) cuando la radiación solar fue alta. Andresen et al. (2010) determinaron en el laboratorio que esta especie se aclimata a radiaciones altas, sin presentar inhibición en su crecimiento, lo cual es característico de las aguas en regiones tropicales. La capacidad de esta especie de variar las concentraciones de ficobilinas, carotenoides y otros pigmentos fotosintéticos le permite que prolifere en intensidades de luz altas y bajas. En Costa Rica, T. erythraeum solo se había presentado formando FAN en el 2002, acompañada por una sola especie (C. polykrikoides) (Vargas-Montero et al. 2008), mientras que la FAN reportada en este estudio fue acompañada por 9 especies.

En décadas recientes, las floraciones de Cochlodinium son uno de los principales problemas de FAN. $C$. polykrikoides, considerado sinónimo de C. catenatum (Matsuoka et al. 2008), tiene una amplia distribución en aguas tropicales y cálidas: Mar Caribe, este y oeste del Océano Pacífico, este del Océano Atlántico, Océano Índico, Mar Mediterráneo, en aguas costeras templadas del noreste de EE. UU., Columbia Británica y oeste de Canadá (Richlen et al. 2010).

De las especies descritas previamente por Hargraves \& Víquez (1985), solamente C. polykrikoides produjo FAN en el golfo durante el periodo 2008-2010, las floraciones de esta especie causan aguas rojizas con espuma y mucus en abundancia. Además, se percibe un olor dulce muy penetrante que provocó náuseas y malestares en las personas (especialmente niños) de las comunidades costeras (Calvo, 2012), se han registrado concentraciones tan altas como $8 \times 10^{7}$ Cél L $^{-1}$ (Hargraves \& Víquez, 1981) y $7.5 \times 10^{7}$ Cél L ${ }^{-1}$ (Calvo et al., en preparación).

Los resultados obtenidos en este estudio coinciden con los datos de Richlen et al. (2010) sobre una floración de octubre de 2008 en el golfo Arábigo, donde C. polykrikoides mostró una concentración de $2.1 \mathrm{x}$ $10^{7} \mathrm{Cé}^{-1} \mathrm{~L}^{-1}$ en la superficie a $27^{\circ} \mathrm{C}$ de temperatura y 39 UPS. Por su parte, Cortés-Lara et al. (2004) observaron en Bahía Banderas (México) una FAN de $C$. catenatum a temperaturas de 25 a $32^{\circ} \mathrm{C}$ y salinidades de 33 a 35 UPS. Las grandes abundancias, registradas particularmente en el puerto pesquero de la Cruz de Huanacaxtle, Nayarit, 
indicaron que esta especie estuvo mejor adaptada a esa zona, donde existen bajas intensidades de luz y aporte de nutrientes del río Ameca.

En las aguas costeras de Guatemala, Carrillo et al. (2007) reportaron una floración de $C$. catenatum (mayo-junio de 2004) con un máximo de $9.64 \times 10^{5}$ Cél $\mathrm{L}^{-1}$ y a temperaturas entre $30.3 \mathrm{y}$ $32.7^{\circ} \mathrm{C}$ y salinidades entre 32.1 y 32.8 UPS. Las máximas concentraciones se observaron más en la superficie que a $5 \mathrm{~m}$ de profundidad.

Kudela et al. (2008) mencionaron que las FAN de Cochlodinium se presentan en un rango de temperatura de 18 a $30^{\circ} \mathrm{C} \mathrm{y}$ salinidades de 30 a 35.8 UPS, sugiriendo que es un organismo euritermal $\mathrm{y}$ eurihalino. Chang et al. (1999) observaron la mortalidad de peces provocada durante unaFAN de Cochlodinium spp., y adujeron que este organismo produce radicales $\mathrm{O}_{2}$ (ROS), aniones de superóxidos $\left(\mathrm{O}_{2}^{-}\right)$, hidroxilos $\left(\mathrm{OH}^{-}\right)$y peróxido de hidrógeno $\left(\mathrm{H}_{2} \mathrm{O}_{2}\right)$, causando daños oxidativos en el funcionamiento y estructura de las células branquiales, y reduciendo la capacidad de transferencia de oxígeno. C. polykrikoides se ha registrado en el Golfo de Nicoya en 1979 y 1985, y a lo largo de la costa pacífica de Costa Rica en el 2003 y el 2004, en cuyos eventos ha sido acompañada por Ceratium spp., P. bahamense var. compressum, Prorocentrum spp., Myrionecta rubra, G. catenatum y T. erythraeum (VargasMontero et al. 2008). En este estudio, la FAN de C. polykrikoides ocurrió en el 2010 y estuvo acompañada por 16 especies.

$P$. minimum es un dinoflagelado potencialmente nocivo con una amplia distribución geográfica en aguas templadas y subtropicales (Grzebyk et al. 1997; Berden-Zrimec et al. 2008), en ambientes costeros y estuarinos (Heil et al. 2004; Taş \& Okuş, 2011), bordeando el Pacífico Norte, las costas este y sur de Estados Unidos de Norteamérica, el Atlántico noreste, el Canal Inglés, los mares del Norte, Báltico, Mediterráneo y Negro, y recientemente ha aparecido en aguas templadas del Hemisferio Sur (Uruguay y Nueva Zelanda). Su ocurrencia en aguas tropicales es inusual (costas de Pakistán y Ecuador). Las proliferaciones en general se presentan en zonas con fuentes de agua dulce como deltas, estuarios, fiordos, lagunas y/o entradas antropogénicas (Grzebyk et al. 1997). Algunas cepas pueden producir dos tipos de toxinas (hepatotoxinas y diarreicas), relacionadas con mortalidad de peces y mariscos, y peligrosas para los consumidores de mejillones, especialmente durante una floración (Witek \& Plínski, 2000).

Taş \& Okuş (2011) consideraron a P. minimum potencialmente tóxico para los humanos al consumir moluscos y de impacto nocivo sobre los ecosistemas por causar condiciones de hipoxia o anoxia y muerte de peces. Es una especie euritermal, eurihalina, y se desarrolla cerca de las costas en ambientes ricos en nutrientes, su nutrición es autotrófica y mixotrófica, permitiéndole dominar y formar floraciones en condiciones de limitación de nutrientes.

Durante el periodo de estudio, la FAN de $P$. minimum se registró en el mes más lluvioso, setiembre de 2010, y fue la única observada en la estación interna del golfo (Isla Pajarita). Esta fue 
la primera vez que se ha detectado una floración de esta especie en el Golfo de Nicoya, alcanzando una dominancia de $89.09 \%$ de la comunidad fitoplanctónica, con temperatura de $27.75^{\circ} \mathrm{C}$, salinidad de 23.79 UPS y una concentración de oxígeno disuelto de $7.98 \mathrm{mg} \mathrm{L}^{-1}$.

Berden-Zrimec et al. (2008) mencionan que $P$. minimum puede crecer en un rango amplio de salinidades, pero prefiere salinidades entre 15 y 35 UPS, y Grzebyk \& Berland (1996) la caracterizaron en laboratorio como eurihalina (15-35 UPS) y euritérmica $\left(8-31^{\circ} \mathrm{C}\right)$.

En el estuario "Golden Horn" (Turquía), las FAN de esta especie alcanzaron una dominancia específica de $90 \%$, en temperaturas entre 19.0 y $24.2^{\circ} \mathrm{C}$ y salinidades entre 15.2 y 18.5 UPS, fuerte intensidad de luz, aguas ricas en nutrientes y en condiciones de calma. Antes del estudio, la especie no era conocida en ese estuario, una vez que se observó formó floraciones con una alta periodicidad (Taş \& Okuş, 2011). Por lo tanto, P. minimum posee una versatilidad ecológica por su amplia distribución. La especie puede poseer una ventaja competitiva en ambientes fríos turbios, con aportes de nutrientes desde tierra y ríos (Grzebyk et al. 1997; Gallegos \& Bergstrom, 2005; Berden-Zrimec et al. 2008).

Según Calvo Vargas et al. (2014), el fenómeno atmosférico de El Niño del año 2009 se caracterizó por una estación lluviosa de baja intensidad $\mathrm{y}$ valores altos en la temperatura $\mathrm{y}$ salinidad del agua, contrariamente durante los eventos de La Niña de los años 2008 y 2010, las precipitaciones fueron mayores con temperatura $y$ salinidad más bajas, siendo el 2010 más fuerte que el 2008. Probablemente, esa sea la razón por la cual durante el 2010 se hayan registrado mayor número de FAN, ya que al haber mayor precipitación la entrada de nutrientes al golfo es mayor, las aguas se tornan más frías y menos salinas, condiciones ambientales que favorecieron a algunas especies de microalgas para que proliferaran. Kress et al. (2001) y León et al. (1998-1999) aducen que la hidrología del golfo depende de los patrones de lluvia y vientos. La entrada de nutrientes durante la época lluviosa a este sistema estuarino es a través de los ríos Tempisque y Grande de Tárcoles, lo cual tiene un efecto estimulante sobre la abundancia del fitoplancton y su composición (Calvo, 2002; 2012).

\section{CONCLUSIONES}

Las FAN en el Golfo de Nicoya fueron más frecuentes durante la época lluviosa, probablemente debido a los aportes fluviales al sistema estuarino de los ríos Tempisque, Barranca y Grande de Tárcoles. Esa estacionalidad, en conjunto con las alteraciones provocadas por eventos atmosféricos globales como La Niña y El Niño, determinó la formación de FAN en el golfo durante el periodo de estudio.

Las especies responsables de las floraciones incluyeron a los dinoflagelados A. monilatum, L. fissa, C. polykrikoides, P. minimum, así 
como la diatomea $S$. costatum y la cianobacteria $T$. erythraeum, las cuales son características de zonas eutrofizadas.

La eutrofización, causada por la descarga de aguas residuales y de nutrientes de los estanques de cultivo, podría estar favoreciendo la proliferación de microalgas en el Golfo de Nicoya.

La presencia de otras especies de dinoflagelados sugiere que las condiciones hidrográficas y fisicoquímicas del golfo han cambiado recientemente y/o que se trata de especies introducidas con el desecho de aguas de lastre de los barcos que las transitan.

Las condiciones de hipoxia que causan el decaimiento inmediato de las floraciones algales afectan la calidad del agua marino-costera del Golfo de Nicoya.

$P$. minimum raramente ha sido asociado a toxinas; sin embargo, algunas cepas producen venerupinas y neurotoxinas, por lo que se debe reconsiderarsu latente potencial tóxico, especialmente porque esta especie a menudo prolifera en áreas de cultivos de organismos marinos provocando disminución en el crecimiento $\mathrm{y}$ susceptibilidad a enfermedades.

Los programas de control periódico de las FAN y de los parámetros hidrográficos en el Golfo de Nicoya son necesarios para prevenir y mitigar sus impactos sobre la salud pública y la muerte masiva de organismos marinos, especialmente aquellos de importancia comercial.

\section{AGRADECIMIENTOS}

Los autores agradecen a la Universidad Nacional el apoyo para la realización de este estudio. Una parte de este trabajo fue financiada con fondos de la Ley de Pesca y Acuicultura No. 8436. Al Instituto Meteorológico Nacional de Costa Rica por proveer los datos de precipitación y radiación solar. A Claudio Calvo Vargas por sus observaciones y aportes tan valiosos. Al capitán Orlando Torres Castro por la colaboración en la recolecta de las muestras. Les agradecemos a los revisores anónimos.

\section{BIBLIOGRAFÍA}

Alpermann, T. J., Tillman, U., Beszteri, B., Cembella, A. D. \& Uwe, J. (2010). Phenotypic variation and genotypic diversity in a planktonic population of the toxigenic marine dinoflagellate Alexandrium tamarense (Dinophyceae). $J$. Phycol., 46, 18-32. doi: 10.1111/j.1529-8817.2009.00767.x.

Anderson, D. M., Kulis, D. M., Sullivan, J. J., Hall, S. \& Lee, C. (1990). Dynamics and physiology of saxitoxin production by the dinoflagellates Alexandrium spp. Mar. Biol., 104, 511524. doi: 10.1007/BF01314358.

Anderson, D. M., Cembella, A. D. \& Hallegraeff, G. M. (2012). Progress in understanding harmful algal blooms: paradigm shifts and new technologies for research, monitoring, and management. Annu. Rev. Mar. Sci., 4, 143-176. doi: 10.1146/ annurev-marine-120308-081121.

Andresen, E., Lohscheider, J., Šetlikova, E., Adamska, I., Šimek, M. \& Küpper, H. (2010). Acclimation of Trichodesmium erythraeum 1SM101 to high and low irradiance analysed on the physiological, biophysical and biochemical level. New Phytologist, 185(1), 173-188. doi: 10.1111/j.1469-8137.2009.03068.x. 
Basti, L., Nagai, S., Go, J., Okano, S., Nagai, K., Watanabe, R., ... \& Tanaka, Y. (2015). Differential inimical effects of Alexandrium spp. and Karenia spp. on cleavage, hatching, and two larval stages of Japanese pearl oyster Pinctada fucata martensii. Harmful Algae, 43, 1-12. doi: 10.1016/j.hal.2014.12.004.

Berden-Zrimec, M., Flander-Putrle, V., Drinovec, L., Zrimec, A. \& Monti, M. (2008). Growth, delayed fluorescence and pigment composition of four Prorocentum minimum strains growing at two salinities. Biol. Res., 41, 11-23. doi: 10.4067/ S0716-97602008000100003.

Calvo, E. (2002). Factores bióticos $y$ abióticos relacionados con la distribución del dinoflagelado tóxico Pyrodinium bahamense var. compressum (Plate 1906) en el Golfo de Nicoya, Costa Rica. Tesis de Licenciatura no publicada, Universidad Nacional, Heredia, Costa Rica.

Calvo, E., Víquez, R. \& García, A. (2005). Alexandrium monilatum (Howell) Balech Bloom in the Gulf of Nicoya, Puntarenas. In The Intergovernmental Oceanographic Commission of UNESCO (Eds.), Harmful Algae News, 29, 1-2.

Calvo, E. (2012). Comportamiento del microfitoplancton marino en el Golfo de Nicoya, Costa Rica: Variación estacional, floraciones nocivas y efectos de El Niño y La Niña durante 2008 a 2010. Tesis de Maestría no publicada, Universidad Nacional, Heredia, Costa Rica.

Calvo Vargas, E., Boza Abarca, J. \& Berrocal Artavia, K. (2014). Efectos de El Niño y La Niña sobre el comportamiento del microfitoplancton marino y las variables fisicoquímicas durante el 2008 a 2010 en el Golfo de Nicoya, Costa Rica. Rev. Mar.
Cost., 6, 115-133. doi: http://dx.doi. org/10.15359/revmar.6.8.

Carrillo, O. L., Cortés-Altamirano, R., Leiva-Cerezo, V. \& Ramírez, V. (2007). Cochlodinium catenatum on Guatemala coast (2004 \& 2007). In The Intergovernmental Oceanographic Commission of UNESCO (Eds.), Harmful Algae News, 33, 15-17.

Carvalho, M., Gianesella, S. M. F. \& Saldanha-Corrêa, F. M. P. (2008). Trichodesmium erythraeum Bloom on the Continental Shelf off Santos, Southeast Brazil. Braz. J. Oceanogr., 56(4), 307-311. doi: 10.1590/S1679-87592008000400006.

Castellví, J. (1963). Pigmentos de la diatomea marina Skeletonema costatum (Grev.) en su dependencia de los factores ambientales y de la dinámica de las poblaciones. Inv. Pesq., 24, 129-137.

Castro, E. \& León, S. (1998). El estado del arte de la investigación en valoración de aguas degradadas en regiones marino-costeras: El caso del Golfo de Nicoya. Informe Preliminar. Centro Internacional de Política Económica para el Desarrollo Sostenible-CINPE, Heredia, Costa Rica.

Chang, S. K., Lee, S. G., Lee, C. K., Kim, H. G. \& Jung, J. (1999). Reactive oxygen species as causative agents in the icthyotoxicity of red tide dinoflagellate Cochlodinium polykrikoides. J. Plankton Res., 21(11), 2105-2115. doi: 10.1093/plankt/21.11.2105.

Choi, J. K., Min, J. E., Noh, J. H., Han, T. H., Yoon, S., Park, Y. J., ... \& Park, J. H. (2014). Harmful algal bloom (HAB) in the East Sea identified by the Geostationary Ocean Color Imager (GOCI). Harmful Algae, 39, 295-302. doi: 10.1016/j.hal.2014.08.010. 
Cortés-Lara, M. C., Cortés-Altamirano, R. \& Sierra-Beltrán, A. P. (2004). Presencia de Cochlodinium catenatum (Gymnodiniales: Gymnodiniaceae) en mareas rojas de Bahía Banderas, Pacífico mexicano. Rev. Biol. Trop., 52(Suppl. 1), 35-49.

Da-Zhi, W., Shu-Gang, Z., Hai-Feng, G., Leo, C. \& Hua-Sheng, H. (2006). Paralytic shellfish toxin profiles and toxin variability of the genus Alexandrium (Dinophyceae) isolated from the southeast China Sea. Toxicon, 48(2), 138-151. doi: 10.1016/j. toxicon.2006.04.002.

Gallegos, C. L. \& Bergstrom, P. W. (2005).

Effect of a Prorocentrum minimum bloom on light availability for and potential impacts on submersed aquatic vegetation in upper Chesapeake Bay. Harmful Algae, 4, 553-574. doi: 10.1016/j.hal.2004.08.016 .

Gárate-Lizárraga, I., López-Cortés, D. J., Bustillos-Guzmán, J. J. \& FernándezSandoval, F. (2004). Blooms of Cochlodinium polykrikoides (Gymnodiniaceae) in the Gulf of California, Mexico. Rev. Biol. Trop., 52(Suppl. 1), 51-58.

Gárate-Lizárraga, I., Muñetón-Gómez, M. \& Maldonado-López, V. (2006). Florecimiento del dinoflagelado Gonyaulax polygramma frente a la Isla Espíritu Santo, Golfo de California, México. Rev. Invest. Mar., 27(1), 31-39. Gárate-Lizárraga, I. \& Muciño-Márquez, R. E. (2012). Blooms of Trichodesmium erythraeum and T. thiebautii (Cyanobacteria, Oscillatoriales) in the Bahía de La Paz, Gulf of California. Oceánides, 27(1), 61-64.

Gárate-Lizárraga, I., Sevilla-Torres, G., Álvarez-Añorve, M., AguirreBahena, F., Violante-González, J.
\& Rojas-Herrera, A. (2013). First record of a red tide caused by Gyrodinium instriatum (Dynophyceae: Gymnodiniales) in Bahía de Acapulco, Guerrero. Oceánides, 28(1), 43-47.

Gárate-Lizárraga, I. (2013). Bloom of Cochlodinium polykrikoides (Dynophyceae: Gymnodiniales) in Bahía de La Paz, Gulf of California. Mar. Pollut. Bull., 67, 217-222. doi: 10.1016/j.marpolbul.2012.11.031.

Gárate-Lizárraga, I. (2014). Proliferation of Levanderina fissa and Polykrikos hartmannii (Dinophyceae: Gymnodiniales) in Bahía de La Paz, Gulf of California, México. Oceánides, 29(2), 25-35.

Grzebyk, D. \& Berland, B. (1996). Influences of temperature, salinity and irradiance on growth of Prorocentrum minimum (Dinophyceae) from Mediterranean Sea. J. Plankton Res., 18(10), 1837-1849. doi: 10.1093/ plankt/18.10.1837.

Grzebyk, D., Denardou, A., Berland, B. \& Pouchus, Y. F. (1997). Evidence of a new toxin in the red-tide dinoflagellate Prorocentrum minimum. J. Plankton Res., 19(8), 1111-1124. doi: 10.1093/ plankt/19.8.1111.

Hansen, G. \& Moestrup, Ø. (1998). Fine-structural Characterization of Alexandrium catenella (Dinophyceae) with special emphasis on the flagellar apparatus. Eur. J. Phycol., 33, 281-291.

Hargraves, P. \& Víquez, R. (1981). The dinoflagellate red tide in Golfo of Nicoya, Costa Rica. Rev. Biol. Trop., 29(1), 31-38.

Hargraves, P. \& Víquez, R. (1985). Spatial and temporal distribution of phytoplankton in the Gulf of Nicoya, Costa Rica. Bull. Mar. Sci., 37(2), 577-585.

Heil, C., Glibert, P. \& Fan, C. (2004). Prorocentrum minimum (Pavillard) 
Schiller A review of a harmful algal bloom species of growing worldwide importance. Harmful Algae, 4(3), 449470. doi: 10.1016/j.hal.2004.08.003.

Huo, W. Y. \& Shu, J. J. (2005, noviembre). Outbreak of Skeletonema costatum bloom and its relations to environmental factors in Jiaozhou Bay, China. Ponencia presentada in el WSEAS International Conference on Environment, Ecosystems and Development, Venecia, Italia.

Ibelings, B. W., Backer, L. C., Kardinaal, W. E. A. \& Chorus, I. (2014). Current approaches to cyanotoxin risk assessment and risk management around the globe. Harmful Algae, 40, 63-74. doi: 10.1016/j.hal.2014.10.002.

Juhl, A. R. (2005). Growth rates and elemental composition of Alexandrium monilatum, a red-tide dinoflagellate. Harmful Algae, 4, 287-295. doi: 10.1016/j.hal.2004.05.003.

Kranz, S. A., Levitan, O., Richter, K., Prášil, O., Berman, I. \& Björn, R. (2010). Combined effects of $\mathrm{CO}_{2}$ and light on the $\mathrm{N}_{2}$-fixing cyanobacterium Trichodesmium IMS101: physiological responses. Plant Physiol., 154, 334345. doi: 10.1104/pp.110.159145.

Kress, N., León, S., Brenes, C., Brenner, S. \& Arroyo, G. (2001). Horizontal transport and seasonal distribution of nutrients, dissolved oxygen and chlorophyll- $a$ in the Gulf of Nicoya, Costa Rica: a tropical estuary. Cont. Shelf Res., 763, 1-16.

Kudela, R. M., Ryan, J. P., Blakely, M. D., Lane, J. Q. \& Peterson, T. D. (2008). Linking the physiology and ecology of Cochlodinium to better understand harmful algal bloom events: A comparative approach. Harmful Algae, 7, 278-292. doi: 10.1016/j.hal.2007.12.016.
León, S., Kress, N., Brenes, C. \& Brenner, S. (1998-1999). Una contribución a la ecología del Golfo de Nicoya. Uniciencia, 15-16, 35-37.

Lim, P. T. \& Ogata, T. (2005). Salinity effect on growth and toxin production of four tropical Alexandrium species (Dinophyceae). Toxicon, 45, 699-710. doi: 10.1016/j.toxicon.2005.01.007.

Matsuoka, K., Iwataki, M. \& Kawami, K. (2008). Morphology and taxonomy of chain-forming species of the genus Cochlodinium (Dinophyceae). Harmful Algae, 7, 261-270. doi: 10.1016/j.hal.2007.12.002.

May, S. P., Burkholder, J. M., Shumway, S. E., Hégaret, H. \& Wikfors, G. H. (2010). Effects of the toxic dinoflagellate Alexandrium monilatum on survival, grazing and behavioral response of three ecologically important bivalve molluscs. Harmful Algae, 9, 281-293. doi: 10.1016/j.hal.2009.11.005.

Nagasoe, S., Kim, D., Shimasaki, Y., Oshima, Y., Yamaguchi, M. \& Honjo, T. (2006). Effects of temperature, salinity and irradiance on the growth of the red tide dinoflagellate Gyrodinium instriatum Freudenthal et Lee. Harmful Algae, 5, 20-25. doi: 10.1016/j.hal.2005.06.001.

Nagasoe, S., Shikata, T., Yamasaki, Y., Matsubara, T., Shimasaki, Y., Oshima, Y. \& Honjo, T. (2010). Effects of nutrients on growth of the red-tide dinoflagellate Gyrodinium instriatum Freudenthal et Lee and a possible link to blooms of this species. Hidrobiologia, 651, 225-238. doi: 10.1007/s10750-010-0301-0.

Narayana, S., Chitra, J., Tapase, S. R., Thamke, V., Karthick, P., Ramesh, Ch., ... Mohanraju, R. (2014). Toxicity studies of Trichodesmium erythraeum (Ehrenberg, 1830) bloom extracts, from Phoenix Bay, Port Blair, Andamans. 
Harmful Algae, 40, 34-39. doi: 10.1016/j.hal.2014.10.003.

Negri, A., Bunter, O., Jones, B. \& Llewellyn, L. (2004). Effects of the bloom-forming alga Trichodesmium erythraeum on the pearl oyster Pinctada maxima. Aquaculture, 232, 91-102. doi: 10.1016/S0044-8486(03)00487-3.

Peña, V. \& Pinilla, G. A. (2002). Composición, distribución y abundancia de la comunidad fitoplanctónica de la ensenada de Utría, Pacífico colombiano. Rev. Biol. Mar. Oceanogr., 37(1), 67-81.

Richlen, M. L., Morton, S. L., Jamali, E. A., Rajan, A. \& Anderson, D. M. (2010). The catastrophic 2008-2009 red tide in the Arabian gulf region, with observations on the identification and phylogeny of the fish-killing dinoflagellate Cochlodinium polykrikoides. Harmful Algae, 9, 163172. doi: 10.1016/j.hal.2009.08.013.

Sarno, D., Kooistra, W.H.C.F., Balzano, S., Hargraves, P. E. \& Zingone, A. (2007). Diversity in the genus Skeletonema (Bacillariophyceae): III. Phylogenetic position and morphological variability of Skeletonema costatum and Skeletonema grevillei, with the description of Skeletonema ardens sp. nov. J. Phycol., 43, 156-170. doi: 10.1111/j.1529-8817.2006.00305.x.

Satpathy, K. K., Mohanty, A. K., Sahu, G., Prasad, M. V. R., Venkatesan, R., Natesan, U. \& Rajan, M. (2007). On the occurrence of Trichodesmium erythraeum (Ehr) bloom in the coastal waters of Kalpakkam, east coast of India. Indian J. Sci. Technol., 1(2), 1-9. Siqueira, A., Kolm, H. E. \& Pereira, F. (2006). Offshore distribution patterns of the cyanobacterium Trichodesmium erythraeum ehrenberg and associated phyto- and bacterioplankton in the southern Atlantic coast (Paraná, Brazil). Braz. Arch. Biol. Technol., 49(2), 323-337. doi: 10.1590/ S1516-89132006000300018.

SPSS. (2006). Statistical Package for the social science for Windows, version 17.0. Chicago, EE. UU.: SPSS Inc.

Taş, S. \& Okuş, E. (2011). A review on the bloom dynamics of a harmful dinoflagellate Prorocentrum minimum in the Golden Horn Estuary. Turkish J. Fish. Aquat. Sci., 11, 673-681.

Taylor, J. D., Kegel, J. U., Lewis, J. M. \& Medlin, L. K. (2014). Validation of the detection of Alexandrium species using specific RNA probes tested in a microarray format: Calibration of signal using variability of RNA content with environmental conditions. Harmful Algae, 37, 17-27. doi: 10.1016/j.hal.2014.05.001.

Thillairajasekar, K., Duraipandivan, V., Perumal, P. \& Ignacimuthu, S. (2009). Antimicrobial activy of Trichodesmium erythraeum (Ehr) (microalga) from south east coast of Tamil Nadu, India. Int. J. Integr. Biol., 5(3), 167-170.

Uchida, T., Kamiyama, T. \& Matsuyama, Y.(1997). Predation by a photosynthetic dinoflagellate Gyrodinium instriatum on loricated ciliates. J. Plankton Res., 19(5), 603-608. doi: 10.1093/ plankt/19.5.603.

Vargas-Montero, M., Freer, E., JiménezMontealegre, R. \& Guzmán, J. C. (2006). Occurrence and predominance of the fish killer Cochlodinium polykrikoides onthe Pacific coast of Costa Rica. $S$. Afr. J. Marine Sci., 28(2), 215-217. doi: 10.2989/18142320609504150.

Vargas-Montero, M., Freer, E., Guzmán, J. C. \& Vargas, J. C. (2008). Florecimientos de dinoflagelados nocivos en 
la costa pacífica de Costa Rica. Hidrobiológica, 18(1 Supl.), 15-23.

Víquez, R. \& Hargraves, P. E. (1995). Annual Cycle of Potentially Harmfull Dinoflagellates in the Golfo de Nicoya, Costa Rica. Bull. Mar. Sci., 57(2), 467-475.

Witek, B. \& Plínski, M. (2000). The first recorded bloom of Prorocentrum minimum (Pavillard) Schiller in the coastal zone of the Gulf of Gdańsk. Oceanologia, 42(1), 29-36.

Yamaguchi, K., Oshima, Y., Oda, T. \& Hongo, T. (2011). Cell contact-dependent lethal effect of the dinoflagellate Heterocapsa circularisquama on phytoplanktonphytoplankton interactions. J. Sea Res., 65, 76-83. doi: 10.1016/j. seares.2010.07.006.

Yamasaki, Y., Shikata, T., Nukata, A., Ichiki, S., Nagasoe, S., Matsubara, T.,
.. \& Honjo, T. (2009). Extracellular polysaccharide-protein complexes of a harm alga mediate the allelopathic control it exerts within the phytoplankton community. ISME Journal, 3, 808-817. doi: 10.1038/ismej.2009.24.

Yamasaki, Y., Ohmichi, Y., Shikata, T., Hirose, M., Shimasaki, Y., Oshima, Y. \& Honjo, T. (2010). Species-specific allelopathic effects of the diatom Skeletonema costatum. Thalassas, 27(1), 21-32.

Zhuang, Y., Zhang, H., Hannick, L. \& Lin, S. (2015). Metatranscriptome profiling reveals Versatile $\mathrm{N}$-nutrient utilization, $\mathrm{CO}_{2}$ limitation, oxidative stress, and active Toxin production in an Alexandrium fundyense bloom. Harmful Algae, 42, 60-70. doi: 10.1016/j.hal.2014.12.006. 